\title{
GENERACIONES FUTURAS Y POLÍTICA A LARGO PLAZO: UN ESTUDIO SOBRE LA LEY PARA EL BIENESTAR DE LAS GENERACIONES FUTURAS DE GALES
}

\section{FUTURE GENERATIONS AND LONG-TERM POLITICS: A STUDY ON THE WELL-BEING OF FUTURE GENERATIONS (WALES) ACT}

\author{
Marcos de ARMENTERAs CABOt \\ Universitat Rovira i Virgili \\ marcos.dearmenteras@urv.cat
}

Fecha de recepción: 15 de abril de 2021 / Fecha de aceptación: 17 de mayo de 2021

RESUMEN: En el año 2015 la Asamblea Nacional de Gales aprobó la Ley para el Bienestar de las Generaciones Futuras. Esta norma, en vigor desde el año 2016, cuyo objeto es garantizar el bienestar de las generaciones futuras, instituyó el Comisionado para las Generaciones Futuras de Gales como órgano encargado de supervisar y auditar que las administraciones públicas orientaran sus políticas hacia unos objetivos de bienestar previamente acordados y fundamentados en el principio de desarrollo sostenible. En el cuarto año de su instauración, el Comisionado ha publicado su primer informe de evaluación (2020) sobre el cumplimiento de los objetivos de bienestar de las generaciones futuras. Este trabajo pretende analizar la ley galesa para el bienestar de las generaciones futuras, el Comisionado, y las posibilidades que abre este tipo de instituciones para la salvaguarda de los intereses de las generaciones futuras y atenuar los problemas vinculados con el cortoplacismo político.

RESUM: L'any 2015 l'Assemblea Nacional de Gal-les va aprovar la Llei pel Benestar de les Generacions Futures. Aquesta norma, en vigor des de l'any 2016, que té com a objectiu garantir el benestar de les generacions futures, va 
instituir el Comissionat per a les Generacions Futures de Gal-les com a òrgan encarregat de supervisar i auditar que les administracions públiques orientessin les seves polítiques cap a uns objectius de benestar prèviament acordats i fonamentats en el principi de desenvolupament sostenible. En el quart any de la seva instauració, el Comissionat ha publicat el seu primer informe d'avaluació (2020) sobre el compliment dels objectius de benestar de les generacions futures. Aquest treball pretén analitzar la llei gal-lesa per al benestar de les generacions futures, el Comissionat, i les possibilitats que obre aquest tipus d'institucions per a la salvaguarda dels interessos de les generacions futures i la atenuació dels problemes vinculats amb el curtterminisme polític.

ABSTRACT: In 2015, the National Assembly for Wales passed the Future Generations Welfare Act. The Act, in force since 2016, whose objective is to guarantee the well-being of future generations, established the Commissioner for Future Generations of Wales as the body in charge of supervising and auditing that public administrations orient their policies towards previously well-being objectives agreed and based on the principle of sustainable development. In the fourth year of its establishment, the Commissioner has published its first evaluation report (2020) on the fulfilment of the welfare objectives of future generations. This article aims to analyse Welsh law for the welfare of future generations, the Commissioner, and the possibilities that this type of institution opens up to safeguard the interests of future generations and mitigate the problems associated with political short-termism.

PALABRAS CLAVE: Instituciones para las generaciones futuras - cortoplacismo político - justicia intergeneracional - desarrollo sostenible - bienestar intergeneracional.

PARAULES CLAU: Institucions per a les generacions futures - curtterminisme polític - justícia intergeneracional - desenvolupament sostenible - benestar intergeneracional.

KEY WORDS: Institutions for future generations - political short-termism intergenerational justice - sustainable development - intergenerational well-being. 
SUMARIO: I. Introducción II. Generaciones futuras, cortoplacismo político y diseño institucional. III. Ley de Gales sobre el bienestar de las generaciones futuras. IV. Análisis y conclusiones. V. Bibliografía.

\begin{abstract}
"I'm not sure, though, what "for later" means anymore. Something changed in the world. Not too long ago, it changed, and we know it. We don't know how to explain it yet, but I think we all can feel it, somewhere deep in our gut or in our brain circuits. We feel time differently. No one has quite been able to capture what is happening or say why. Perhaps it's just that we sense an absence of future, because the present has become too overwhelming, so the future has become unimaginable. And without future, time feels like only an accumulation. An accumulation of months, days, natural disasters, television series, terrorist attacks, divorces, mass migrations, birthdays, photographs, sunrises. We haven't understood the exact way we are now experiencing time"1.
\end{abstract}

\title{
I. INTRODUCCIÓN
}

Los fundamentos de la responsabilidad entre generaciones han sido ampliamente discutidos en las últimas décadas desde diferentes ámbitos del conocimiento $^{2}$. A pesar de este acervo, la tendencia a olvidar las políticas a largo plazo es común en las democracias representativas, lastrando así la capacidad de llevar a cabo políticas constantes a lo largo del tiempo y de considerar sus efectos a largo plazo. El cortoplacismo político y la inconsistencia de las políticas públicas a lo largo del tiempo plantean retos de carácter intergeneracional de gran importancia. Las políticas referidas a los sistemas de pensiones, el sistema

\footnotetext{
1 Vid. Luiselli, V., Lost Children Archive, Alfred A. Knopf, 2019, p. 103.

2 Vid. Sikora, R.I., y Barry, B., (Eds.), Obligations to Future Generations, Philadelphia, Temple University Press, 1978; Pontara, G., Ética y generaciones futuras, Ariel, Barcelona, 1996; Tremmel, J.C., (Ed.) Handbook of Intergenerational Justice, Edward Elgar, 2006; Gosseries, A., y Meyer, L. H., (Eds.), Intergenerational justice, Oxford University Press, 2009; Meyer, L. H., Intergenerational justice, Routledge, 2012. Desde el Derecho, véase Brown Weiss, E., In Fairness to Future Generations: International Law, Common Patrimony, and Intergenerational Equity, United Nations University, Tokyo, 1989. Desde la economía política, véase Roemer, J., y Suzumura, K. (Eds.), Intergenerational Equity and Sustainability, Palgrave Macmillan, 2007. Desde el plano ecologista, Riechmann afirma: "En la era industrial, las consecuencias de lo que hacemos (y dejamos de hacer) llegan cada vez más lejos en el espacio y en el tiempo. Sugiero que, por eso, en términos éticos e históricos podemos caracterizar nuestro tiempo como la época moral del largo alcance". Cfr. Riechmann, J., Un mundo vulnerable, Los Libros de la Catarata, Madrid, 2005 ( $2^{\mathrm{a}}$ ed.), p. 164. En un sentido similar, desde una perspectiva comunitarista, deShalit afirma que la existencia de una comunidad "transgeneracional" en el que las generaciones comparten relaciones de cooperación y en la existencia de ella misma radica la justificación de la responsabilidad intergeneracional, véase, de-Shalit, A., Why Posterity Matters: Environmental Policies and Future Generations, Rouledge, 1995, pp. 13 - 15.
} 
educativo, el cambio climático, los daños ambientales o los sistemas de producción de energía, entre otras, no solo afectarán a los ciudadanos presentes, sino también a las generaciones futuras. Así, a pesar de que los segmentos más jóvenes de las generaciones presentes y las generaciones futuras cargarán con el peso de aquellas decisiones actuales, los efectos a largo plazo de las políticas no siempre son atendidos en el proceso de toma de decisiones. Es por ello que resulta de interés dar cuenta de las disfunciones producidas por la ausencia de un análisis prospectivo de las políticas públicas y analizar los mecanismos que podrían ayudar a paliar sus efectos.

Para ello, en las últimas décadas, se han propuesto diferentes mecanismos que podrían ayudar a atenuar los problemas derivados del cortoplacismo político y la inconsistencia de las políticas a lo largo del tiempo. La creación de organismos independientes para la salvaguarda los intereses de las generaciones futuras ocupa un lugar destacado.

En 1988, el Comité Asesor establecido para el Proyecto sobre "Derecho internacional, patrimonio común y equidad intergeneracional" de la Universidad de Naciones Unidas, presentó una propuesta de estrategia para implementar "Ios derechos y obligaciones intergeneracionales". Entre otras propuestas, el Comité recomendó la designación de defensores o comisionados para proteger los intereses de las generaciones futuras ${ }^{3}$. Desde entonces un buen número de Estados y regiones han creado organismos independientes que llevan a cabo este cometido. Los más destacados son el Defensor de las Generaciones Futuras de Hungría, el Guardián de las Generaciones futuras de Malta, la Comisión Parlamentaria en Israel -ahora ya inactiva-, el Comité para el Futuro de Finlandia; o aquellos especializados en cuestiones ambientales, el Consejo Asesor para el Desarrollo Sostenible del Parlamento alemán, el Comisionado para el Medio Ambiente y el Desarrollo Sostenible de Canadá, el Comisionado Parlamentario para el Medio Ambiente de Nueva Zelanda, o el Comisionado para la Sostenibilidad y el Medio Ambiente de Australia.

\footnotetext{
${ }^{3}$ Cfr. "The Goa Guidelines on Intergenerational Equity", 1988 (Goa, India).
} 
En este contexto, La Asamblea Nacional de Gales aprobó en el año 2015 la Ley sobre el Bienestar de las Generaciones Futuras ${ }^{4}$, en la que marcaba unas pautas de actuación para que las autoridades públicas tuvieran en cuenta los impactos a largo plazo en su toma de decisiones y estableció un organismo encargado de auditar la actividad de las autoridades públicas en relación con la salvaguarda del bienestar de las generaciones futuras.

Esta contribución explora las oportunidades que la ley galesa para el bienestar de las generaciones futuras presenta para corregir los problemas del cortoplacismo político. En primer lugar, daré cuenta de las causas del cortoplacismo político y las distintas propuestas para atenuarlo. En segundo lugar, analizaré la ley que instituye el Comisionado, y analizaré sus funciones y obligaciones. Para concluir, analizaré las oportunidades y limitaciones de la ley y el organismo que instituye.

\section{GENERACIONES FUTURAS, CORTOPLACISMO POLÍTICO Y DISEÑO INSTITUCIONAL}

A pesar de que es difícil dar cuenta de un listado cerrado de cuestiones que deben ser analizadas a partir de estrategias a largo plazo, sí que podemos señalar un buen número de asuntos que tienen un gran impacto en el medio y largo plazo, como el cuidado del medio ambiente, el cambio climático, la inversión en infraestructuras, en educación, en investigación científica o en el sistema de pensiones. Estas cuestiones, entre otras, determinarán el grado de bienestar del que disfrutarán las generaciones presentes y futuras a lo largo de su vida a medio y largo plazo. La ausencia de políticas prospectivas limitará la capacidad de responder en el futuro a aquellas cuestiones que requieren políticas constantes y a largo plazo, impidiendo incorporar escenarios deseados y reduciendo los riesgos en el futuro.

En este sentido, para un correcto análisis es oportuno preguntarse en qué fallan los sistemas representativos al incorporar perspectivas a largo plazo y cómo es posible orientar las políticas públicas en términos intergeneracionales para evitar

\footnotetext{
4 "Well-being of Futures Generations Act", aprobada por la Asamblea Nacional de Gales y formalmente promulgada por Consentimiento Real el 29 de abril de 2015. En vigor desde el 22 de julio de 2016. [Disponible aquí: https://www.futuregenerations.wales/wpcontent/uploads/2017/01/WFGAct-English.pdf. Última consulta el 20 de marzo de 2021].
} 
el cortoplacismo político en aquellas cuestiones que lo requieran. Para ello, en primer lugar, analizaré el cortoplacismo político y cómo el diseño de instituciones para las generaciones futuras puede servir para avanzar en una mejor integración de los intereses de las generaciones futuras en la política del presente.

\section{Cortoplacismo político: causas}

El cortoplacismo político es un problema inherente a las democracias representativas contemporáneas ${ }^{5}$. La literatura especializada enfatiza diferentes causas que llevan a los representantes políticos y a los ciudadanos a favorecer las políticas a corto plazo sobre aquellas a medio y largo plazo. Parece intuitivo que los ciudadanos y sus representantes podrían restar valor a aquellas políticas beneficiosas a largo plazo, y otorgarán preferencia a aquellas cuyo resultado pueda ser alcanzado a corto plazo. Igualmente, las políticas que tengan un coste en el presente y un beneficio en el futuro, podrían causar un alto rechazo ciudadano. Por ejemplo, la substitución de fuentes de energía dependientes de combustibles fósiles por dispositivos capaces de generar energía desde fuentes renovables supone una inversión en el presente para el desarrollo tecnológico, la substitución de fuentes de energía o la electrificación del sistema energético que impactará positivamente en el futuro en términos de reducción de emisiones de gases de efecto invernadero y mayor seguridad en el suministro energético; igualmente, la inversión en investigación científica requiere de una inversión presente que sea continua en el tiempo para poder obtener los beneficios derivados de ésta en el futuro; o la inversión en un sistema de pensiones sostenible es beneficioso a largo plazo porque ayuda a mejorar las condiciones de vida de los pensionistas sin situar toda la carga en las generaciones futuras.

\footnotetext{
${ }^{5}$ El politólogo D. Thompson, lo denomina "miopía democrática hacia el futuro" y destaca cuatro razones que según él las democracias olvidan la política a largo plazo: 1) la tendencia humana a preferir el beneficio inmediato, sesgo que también reproducen los políticos en su ámbito de acción; (2) los representantes tienen en consideración las opiniones de los ciudadanos y sobre los efectos que las leyes y políticas que llevan a cabo tienen sobre ellos; (3) los gobernantes ejercen el poder durante un tiempo limitado y ello conlleva un cierto interés en que sus políticas tengan efecto en un periodo de tiempo; y (4) en la mayoría de democracias hay una tendencia a proteger los intereses de los grupos de edad avanzada sobre los intereses de los grupos de menor edad por razones, principalmente, electorales. Vid. Thompson, D. F., "Representing future generations: political presentism and democratic trusteeship", Critical Review of International Social and Political Philosophy, 13 (1), 2010, pp. 3-5.
} 
En este sentido, antes de examinar el Comisionado de Gales resulta relevante dar cuenta de las causas del cortoplacismo político para así poder comprender sus deficiencias y potencialidades. Así, González-Ricoy, analizando las capacidades de diferentes instituciones "intergeneracionales" para responder al cortoplacismo político, a través de estudios empíricos y normativos, da cuenta de sus causas y las clasifica en tres determinantes: motivacionales, epistémicos e institucionales ${ }^{6}$. Los primeros serían aquellos por los que los ciudadanos restarían valor a las políticas a largo plazo y otorgarían mayor valor a aquellas llevadas a cabo a corto plazo; igualmente lo representantes públicos favorecerían las políticas a corto plazo por alcanzar beneficios electorales, resultados palpables de su gestión, etcétera. Los segundos estarían vinculados con la falta de información y conocimiento de los ciudadanos presentes sobre aquello que podrá ocurrir en el futuro, y sobre los sesgos temporales característicos del ser humano. La incertidumbre, en este caso, sería determinante para que los ciudadanos actuales descontaran valor a los beneficios que se pudieran lograr en el futuro ${ }^{7}$. Los terceros estarían vinculados, por un lado, con aquellos problemas de debilidad de las instituciones, ya sea debido a incapacidades propias para aplicar medidas que conlleven incorporar recursos públicos del presente para un beneficio futuro; o debido a que la percepción de corrupción o ineficacia de las instituciones, lleva a los ciudadanos a desconfiar de aquellas políticas sobre las que no pueden ejercer un control más riguroso. Por otro lado, los determinantes institucionales, estarían vinculados con problemas relacionados con la coordinación de dichas políticas debido, entre otras causas, a los cambios de gobierno y consecuentes cambio de orientación en las políticas públicas, o las situaciones de alta tensión política ${ }^{8}$.

\section{Diseño institucional y políticas a largo plazo}

Para dar respuesta a estos problemas de cortoplacismo político, diferentes tipos de mecanismos intergeneracionales han sido discutidos y propuestos a lo largo

\footnotetext{
6 Vid. González-Ricoy, I., "Instituciones intergeneracionales y cortoplacismo político", Revista Española de Ciencia Política, 41, 2016, pp. 67-92. A este respecto, González-Ricoy también cita el trabajo de Thompson (n. 10). Además, Thompson indica: "Most citizens tend to discount the future, and to the extent that the democratic process responds to their demands, the laws it produces tend to neglect future generations. The democratic process itself amplifies this natural human tendency". Vid. Thompson, D. F., (n. 10), p. 17.

7 Ibid. p. 80.

8 Ibid. p. $82-83$.
} 
de los últimos años ${ }^{9}$. A este respecto podríamos clasificar los mecanismos en dos modelos. Uno, que aglutina aquellas herramientas o modificaciones institucionales que favorecerían la toma de decisiones en las instituciones públicas ya existentes, y otro, que se concretaría a partir de la creación de organismos independientes para auditar la actividad política en términos intergeneracionales.

Entre el primer modelo encontraríamos aquellos instrumentos para medir nuestro impacto intergeneracional, en el campo ambiental destacan la "huella ecológica" y la "biocapacidad"10, o indicadores que abarcan más ámbitos como los incorporados para evaluar los Objetivos de Desarrollo Sostenible ${ }^{11}$. También nos encontramos con aquellas que permitirían la inclusión de forma directa a las generaciones futuras en nuestro proceso de toma de decisiones, como cuotas de jóvenes en los parlamentos ${ }^{12}$, representantes de entidades ecologistas ${ }^{13}$; 0 representantes de las generaciones futuras ${ }^{14}$; o a través del reconocimiento de normas que prohíben la regresión en los avances en términos de derechos humanos, en particular en derechos sociales y ambientales ${ }^{15}$. En este sentido, otro mecanismo planteado sería la constitucionalizarían de ciertos aspectos

\footnotetext{
9 Vid. González-Ricoy, I., y Gosseries, A., (Eds.), Institutions for Future Generations, Oxford University Press, 2016.

10 Vid. Nogueira, A., "La huella ecológica. El establecimiento de indicadores ambientales y su significación para el Derecho", Revista Catalana de Dret Ambiental, Vol. 10 Núm. 1, 2019.

${ }_{11}$ Para el seguimiento de los objetivos de la Agenda 2030 para el Desarrollo Sostenible se diseñaron 232 indicadores que se ocupaban de los distintos ámbitos. En la página web del Instituto Nacional de Estadística de España se pueden examinar. [Disponible aquí: https://www.ine.es/dyngs/ODS/es/index.html. Última consulta el 10 de abril de 2021].

12 Vid. Bidadanure, J., "Youth Quotas, Diversity, and Long-Termism: Can Young People Act as Proxies for Future Generations?", en González-Ricoy, I., y Gosseries, A., (Eds.), Institutions for Future Generations, Oxford University Press, 2016.

13 Vid. Dobson, A., "Representative Democracy and the Environment", en Lafferty, W. M., y Meadowcroft, J., (Eds.), Democracy and the Environment: Problems and Prospects, Cheltenham, Edward Elgar, 1996, pp. 124-39.

${ }^{14}$ Partiendo de la propuesta de Dobson, Ekeli plantea que un $5 \%$ de los escaños parlamentarios sean reservados para los representantes de las generaciones futuras. Vid. Ekeli, K. S., "Giving a Voice to Posterity - Deliberative Democracy and Representation of Future People", Journal of Agricultural and Environmental Ethics 18(5), 2005, pp. 429-450.

${ }^{15}$ En este sentido, el principio de no-regresión es clave. En el ámbito ambiental véase Prieur, M., El nuevo principio de «no regresión» en derecho ambiental, Discurso en la concesión del título de Doctor Honoris Causa de la Universidad de Zaragoza, 2010. En el ámbito de los derechos sociales, véase Añón Roig, M.J., “¿Hay límites a la regresividad de derechos sociales?”, Derechos y Libertades, n. 34, Época II, enero 2016, p. 63; Courtis, C., "La prohibición de regresividad en materia de derechos sociales: apuntes introductorios", en Courtis, C., (Comp.): Ni un paso atrás. La prohibición de regresividad en materia de derechos sociales, Buenos Aires, Centro de Estudios Legales y Sociales (CELS) y Centro de Asesoría Legal del Perú (CEDAL). Ediciones del Puerto, 2006, pp. 48-53.
} 
(como el reconocimiento de los intereses de las generaciones futuras, cláusulas inmanentes, o cláusulas pétreas) ${ }^{16}$.

Entre los segundos encontramos propuestas para la creación de organismos encargados de defender los intereses de las generaciones futuras a través del control y la auditoría de las políticas públicas. Las propuestas son variadas. principal problema vinculado con este tipo de institución es la razón de su misma existencia, su legitimidad para influir en la toma de decisiones en la política presente. Este tipo de instituciones no son de carácter representativo, sino que son instituciones contramayoritarias cuyo cometido es auditar a las instituciones representativas para evitar que caigan en el cortoplacismo político en aquellas políticas que requieren una mirada prospectiva. Entre los diferentes tipos de organismos que ya existen encontramos aquellos que tienen una pretensión más generalista, que abarcan en su cometido diferentes ámbitos de la vida política, como el de Gales, Finlandia o Hungría, y aquellos organismos de carácter sectorial que tienen la función de orientar un aspecto específico de la política, en particular la política ambiental, como las comisiones de Canadá, Nueva Zelanda o Australia.

La incorporación de estas instituciones puede ser objeto de crítica debido a los límites de su legitimidad democrática habida cuenta de su capacidad de influir en las decisiones de las instituciones y administraciones públicas con legitimación democráticas. Así, si los representantes se deben a sus electores, en última instancia, deberían ser los ciudadanos presentes que evalúen si las políticas llevadas a cabo se ajustan a sus intereses y, si no fuera así, los electores deberían tener la posibilidad de substituir a sus representantes por unos que satisfagan sus demandas. Aplicado al caso que nos ocupa, si un

\footnotetext{
${ }^{16}$ Vid. Häberle, P., "Un derecho constitucional para las futuras generaciones. La otra reforma del contrato social: el contrato generacional", Lecciones y Ensayos, Número 87, Enero-Diciembre, 2009. [Traducción de Fellay, M., y Ferreyra, L.]. El autor destaca la inclusión de referencias directas a las generaciones futuras, de cláusulas inmanentes en la Constitución, y cláusulas pétreas. Las cláusulas inmanentes serían aquellas que obligan a la protección de cierto bien normalmente recursos naturales- en beneficio de las generaciones futuras, y las cláusulas pétreas son aquellas que son inderogables. En un sentido similar, Ekeli defiende la implementación de una cláusula hacia la posteridad que estimule a las autoridades a llevar a cabo deliberaciones y decisiones más orientadas al futuro, y concienciar y mejorar el proceso de toma de decisiones de aquellas cuestiones que afecten a las generaciones futuras. Vid. Ekeli, K. S., "Green Constitutionalism: The Constitutional Protection of Future Generations", Ratio Juris, Vol. 20 No. 3, 2007, pp. 378-401.
} 
representante orientara las políticas al largo plazo y estas políticas fueran rechazadas por los electores, éstos acabarían retirando la confianza a dichos representantes y se la concederían a aquellos que llevaran a cabo políticas cortoplacistas. Es precisamente este esquema el que nos trae hasta aquí, la dificultad para orientar políticas a largo plazo y que sean constantes a lo largo del tiempo.

Es un hecho que en las democracias liberales existen mecanismos que limitan la toma de decisiones. En el plano ambiental nos encontramos con principios que obligan a los poderes públicos a actuar preventivamente y precautoriamente ante eventuales daños ambientales. En materia de derechos fundamentales existen límites en los que el legislador en un Estado constitucional no puede decidir ${ }^{17}$. Igualmente, los procesos legislativos, en un gran número de países, están sometidos a sistemas de control constitucionalidad cuya esencia es contramayoritaria y no por ello dejan de ser democráticos ${ }^{18}$. En el plano ecológico, además, es preciso mencionar que reside un problema de carácter intergeneracional que puede poner en cuestión la legitimidad de aquellas decisiones que no tengan en cuenta a las generaciones futuras en el proceso de toma de decisiones. Así, Capella afirma sobre las decisiones vinculadas con la ecología: "[...] ni se puede pensar la democracia sin tener en cuenta a las generaciones futuras"19, y sigue más adelante, "la mayoría debe ser vista críticamente cuando decide cuestiones que indudablemente afectarán a las

\footnotetext{
${ }^{17}$ Así lo expresa Ferrajoli: "Ninguna mayoría, ni siquiera por unanimidad, puede legítimamente decidir la violación de un derecho de libertad o no decidir la satisfacción de un derecho social". Vid. Ferrajoli, L., Derechos y garantías: la ley del más débil, Ed. Trotta, (8ª ed.), 2016, p. 24. [Traducción de lbáñez, P. A., y Greppi, A.]

${ }^{18}$ No voy a entrar en la discusión acerca de la legitimidad de la revisión judicial o constitucional de las normas, pero es conocido que existen desacuerdos sobre la legitimidad del control constitucional. La posición de Waldron en contra de la judicial review seguramente es la más conocida. Vid. Waldron J., Law and Disagreements, New York, Oxford University Press, 1999 p. 15; Posteriormente atenuó un poco su posición entorno la cuestión de la "tiranía de la mayoría" en Waldron, J., "The Core of the Case against Judicial Review", Yale Law Journal, 115, 2006, pp. 1346-1406. Por otro lado, también es cierto que la actividad de los tribunales que deben llevar a cabo esta tarea debe obedecer al principio de presunción de constitucionalidad de las leyes o proceder con deferencia hacia el legislador democrático. Esto lo analiza Ferrerres Comellas en los siguientes trabajos: Ferrerres Comella, V., Constitutional Courts Democratic Values: a European perspective, Yale University Press, New Haven and London, 2009, pp. 88-99; y en Ferrerres Comella, V., Justicia constitucional y democracia, Centro de Estudios Políticos y Constitucionales, (2eed.), 2007, pp. 131-193.

${ }^{19}$ Cfr. Capella, J. R., Un fin del mundo: Constitución y democracia en el cambio de época, Ed. Trotta, Madrid, 2019, p. 80.
} 
generaciones futuras" 20 . No obstante, a pesar de que algunos mecanismos contramayoritarios en los sistemas constitucionales actuales sean comunes, y que planteemos una crítica a las mayorías que deciden asuntos con impacto ambiental intergeneracional, la existencia de un organismo que tenga la capacidad de influir de manera determinante en las decisiones de los representantes en la actualidad puede generar rechazo, pues la ausencia de legitimidad democrática de éstos tensa la relación entre una figura de carácter tecnócrata con gran influencia política, y los legítimos representantes de los ciudadanos.

La fricción entre los órganos representativos y organismos contramayoritarios por la fiscalización de los segundos sobre los primeros puede suscitar cierto rechazo ${ }^{21}$. Así, un organismo que tenga poderes amplios y vinculantes podría causar mayor rechazo y, como consecuencia, ser menos efectivo que otro con poderes más moderados y no vinculantes. El segundo podría recabar mayor aceptación y favorecer a la deliberación pública en torno a los problemas de carácter intergeneracional y lograr mejores resultados. Contrariamente, es posible que los segundos, debido a sus limitaciones pierdan cualquier efectividad y la institución no pueda cumplir sus objetivos. En un equilibrio entre la aceptación social y la efectividad de su actividad se mueven estos organismos.

Los parámetros que podemos seguir para evaluar su capacidad de influencia son, entre otros, la cantidad y el contenido de sus competencias, la vinculatoriedad jurídica de sus resoluciones, la publicidad y auditoría de su gestión, su relación con las diferentes administraciones, o su capacidad para recurrir ante órganos jurisdiccionales o de control de constitucionalidad de las

20 Ibíd., p. 104. Ante el cuestionamiento de la legitimidad de las decisiones en este ámbito resulta de interés las propuestas sobre la idoneidad de las propuestas anteriormente comentadas: incluir cuotas de jóvenes en los parlamentos (Bidadanure, 2016); representantes de las generaciones futuras (Ekeli, 2005); o representantes de entidades ambientalistas (Dobson, 1996).

${ }^{21}$ El caso ya comentado del control de constitucionalidad de las normas por parte de los tribunales constitucionales es el ejemplo paradigmático. La capacidad de un órgano no representativo de limitar las decisiones democráticas no deja de ser problemático. Así, el hecho de que sus decisiones sean de carácter vinculante plantea la necesidad de mantener ciertos equilibrios en su composición, mandato y funcionamiento. Así, que sus miembros sean elegidos a partir órganos representativo, que sus mandatos estén limitados en el tiempo, o que se guíen por ciertos principios, como el de presunción de constitucionalidad de las normas o el de deferencia hacia el legislador (agravada si la norma ha sido sometida a referéndum), son elementos determinantes para que la sociedad legitime un control tan fuerte por una institución contramayoritaria. El abandono o quebranto de este tipo de equilibrios en una institución de este signo podría repercutir en una pérdida de confianza por parte de la ciudadanía. 
normas la legislación aprobada por las instituciones del Estado. En este sentido, la viabilidad de estas instituciones para llevar a cabo políticas a largo plazo y que sean consistentes en el tiempo, irá de la mano en su capacidad de contrarrestar los determinantes anteriormente mencionados y la aceptación social de dicha institución.

\section{LEY DE GALES SOBRE EL BIENESTAR DE LAS GENERACIONES FUTURAS}

Para un adecuado análisis de la norma es preciso dar cuenta del contexto político en el que se aprobó, los objetivos que plantea -que giran en torno al concepto de bienestar y desarrollo sostenible-, y qué tipo de obligaciones generales impone a los organismos públicos para alcancen las metas de bienestar que fundamentan la norma. La ley examinada es extensa y por ello no analizaré todos los extremos de ésta, como las obligaciones específicas que la ley impone a ministros, entes locales y otros organismos públicos.

\section{Origen y desarrollo legislativo.}

El proceso de descentralización del poder llevado a cabo en el Reino Unido desde finales de los años noventa se concretó en Gales a través de la creación de la Asamblea Nacional de Gales en 1998 -que desde mayo de 2020 se denomina Parlamento de Gales ${ }^{22}$-, y se ha ido consolidando en los últimos años a través de la consecución de mayores competencias para su órgano legislativo y una gran actividad legislativa. Así, en virtud de la Ley de Gobierno de Gales (Government of Wales Act 2006), aprobada por el Parlamento del Reino Unido en el año 2006, la Asamblea Nacional de Gales pasaba a tener competencia legislativa en veinte materias específicas ${ }^{23}$. En marzo del año 2011 llevó a cabo

\footnotetext{
${ }^{22}$ La "Senedd and Elections (Wales) Act 2020" modificó el nombre a la Asamblea Nacional por Parlamento. También amplió el derecho a voto pasando el mínimo de edad de los 18 a los 16 años, $y$ a los extranjeros. [Disponible aquí: https://www.legislation.gov.uk/anaw/2020/1/enacted\#: :text=An\%20Act\%20of\%20the\%20Natio nal,Commission\%2C\%20to\%20make\%20miscellaneous\%20changes. Última consulta el 9 de marzo de 2021].

${ }^{23}$ El apéndice quinto de la ley establece las competencias que son las siguientes: agricultura, pesca, silvicultura y desarrollo rural; monumentos y edificios históricos; cultura; desarrollo económico; educación y formación; medio ambiente; servicios de bomberos y rescate y promoción de la seguridad contra incendios; alimentación; salud y servicios sanitarios; carreteras y transporte; vivienda; gobierno local; Asamblea Nacional de Gales; administración pública; bienestar social; deporte y ocio; turismo; planificación urbana y rural; agua y defensa contra inundaciones; y lengua galesa.
} 
un referéndum en el que se preguntaba a la ciudadanía galesa si quería que la Asamblea pudiera legislar sobre los asuntos de las 20 materias en las que tiene competencias $^{24}$. Ante la aprobación por referéndum por más del $60 \%$ de los votos a favor, la siguiente Asamblea, surgida de las elecciones de mayo del mismo año, pasaba a ser una Asamblea con competencias más robustas y con materias de mayor influencia política.

Este aumento de competencias se dio en un contexto en el que el Gobierno galés había llevado a cabo un programa político en el que la sostenibilidad ocupaba un lugar central de su acción política. En el año 2009, en virtud del artículo 79 de la Ley del Gobierno de Gales de $2006^{25}$, el Gobierno publicó su Plan de Desarrollo Sostenible ${ }^{26}$, en el que detallaba un programa pormenorizado presentado como un proyecto político en el que el bienestar de las generaciones presentes y futuras estaba en el centro de acción gubernamental. Para ello, el concepto de desarrollo sostenible se planteaba a partir de acciones concretas a llevar a cabo en términos de sostenibilidad, incluyendo indicadores que tenían en cuenta múltiples objetivos de desarrollo sostenible (ecológicos, económicos, laborales, educativos, etcétera), y especificando las propuestas para cada objetivo. Además, estos objetivos se asentaban en la visión que el Gobierno planteaba para el futuro de Gales ${ }^{27}$. Con este pretexto, en el año 2012, ya con las competencias legislativas adquiridas por la Asamblea, el gobierno comenzó a preparar el Proyecto de Ley de Desarrollo Sostenible que, posteriormente, se

\footnotetext{
${ }^{24}$ La pregunta fue la siguiente: "Do you want the Assembly now to be able to make laws on all matters in the 20 subject areas it has powers for?" [Disponible aquí: https://www.legislation.gov.uk/uksi/2010/2837/article/4/made/data.xht?view=snippet\&wrap=true. Última consulta el 9 de marzo de 2021.

${ }^{25}$ En su artículo 79.1 establece: "The Welsh Ministers must make a scheme ("the sustainable development scheme") setting out how they propose, in the exercise of their functions, to promote sustainable development".

${ }^{26}$ Denominado "One Wales: One Planet the Sustainable Development Scheme of the Welsh Assembly Government", publicado en mayo de 2009. [Disponible aquí: https://www.bridgend.gov.uk/media/1505/wd32.pdf. Última consulta el 9 de marzo de 2021].

${ }^{27}$ Ibíd., p. 17. Establece "Our Vision of a Sustainable Wales is one where Wales: Lives within its environmental limits, using only its fair share of the earth's resources so that our ecological footprint is reduced to the global average availability of resources, and we are resilient to the impacts of climate change; has healthy, biologically diverse and productive ecosystems that are managed sustainably; has a resilient and sustainable economy that is able to develop whilst stabilising, then reducing, its use of natural resources and reducing its contribution to climate change; has communities which are safe, sustainable, and attractive places for people to live and work, where people have access to services, and enjoy good health; is a fair, just and bilingual nation, in which citizens of all ages and backgrounds are empowered to determine their own lives, shape their communities and achieve their full potential".
} 
convirtió en el Proyecto de Ley del Bienestar de las Generaciones Futuras, con el objetivo de que los deberes con respecto a las generaciones futuras constituyeran su marco de acción política ${ }^{28}$. En este sentido, el proyecto presentado inicialmente ya se configuró a partir de la noción de bienestar ${ }^{29}$. El proyecto fue presentado con un memorando en el que se expusieron los beneficios económicos, sociales y ambientales que podría proporcionar su aprobación ${ }^{30}$.

Es preciso mencionar que en el contexto en el que entra en vigor esta norma, también se aprobaron un gran número de normas de carácter ambiental, social y cultural, como la ley de vivienda de $2014^{31}$, la ley de planificación y desarrollo de $2015^{32}$, la ley de violencia contra las mujeres de $2015^{33}$, la ley de educación de $2015^{34}$, la ley ambiental de $2016^{35}$, la ley de monumentos históricos de $2016^{36}$, o la ley de salud pública de $2017^{37}$.

\section{Estructura}

La ley sobre el bienestar de las generaciones futuras está dividida en cinco secciones. La primera es introductoria; la segunda establece los objetivos, las obligaciones sobre los organismos públicos, y los mecanismos para examinar y auditar el cumplimiento de los objetivos de bienestar; la tercera instituye el

\footnotetext{
${ }^{28}$ Vid. Davies, H., "The Well-being of Future Generations (Wales) Act 2015-A Step Change in the Legal Protection of the Interests of Future Generations?", Journal of Environmental Law, 29, 2017, pp. 165-175

${ }^{29}$ Well-Being of Future Generations (Wales) Bill, as introduced, 7 de julio de 2014. [Disponible aquí: https://senedd.wales/media/4msf0o2h/pri-ld9831-e.pdf. Última consulta el 12 de marzo de 2021].

${ }^{30}$ Explanatory Memorandum to the Well-being of Future Generations (Wales) Bill. [Disponible aquí: https://gov.wales/sites/default/files/publications/2021-01/well-being-of-future-generationswales-bill-explanatory-memorandum-march-2015.pdf. Última consulta el 12 de marzo de 2021]. 31 Housing (Wales) Act 2014. [Disponible aquí: https://www.legislation.gov.uk/anaw/2014/7/contents. Última consulta el 10 de marzo de 2021]. 32 Planning (Wales) Act 2015.2 [Disponible aquí: https://www.legislation.gov.uk/anaw/2015/4/contents. Última consulta el 10 de marzo de 2021]. ${ }^{33}$ Violence against Women, Domestic Abuse and Sexual Violence (Wales) Act 2015. [Disponible aquí: https://www.legislation.gov.uk/anaw/2015/3/contents. Última consulta el 10 de marzo de 2021].

34 Higher Education (Wales) Act $2015 . \quad$ [Disponible aquí: https://www.legislation.gov.uk/anaw/2015/1/contents. Última consulta el 10 de marzo de 2021].

35 Environment (Wales) Act 2016. [Disponible aquí: https://www.legislation.gov.uk/anaw/2016/3/contents. Última consulta el 10 de marzo de 2021]. 36 Wales Historic Environment Act 2016. [Disponible aquí: https://www.legislation.gov.uk/anaw/2016/4/contents. Última consulta el 10 de marzo de 2021]. 37 Public Health (Wales) Act 2017. [Disponible aquí: https://www.legislation.gov.uk/anaw/2017/2/contents. Última consulta el 10 de marzo de 2021].
} 
Comisionado para las generaciones futuras y establece su composición y funciones; la cuarta establece los mecanismos de participación a nivel local, su rol en el cumplimiento de las metas de bienestar y los mecanismos para desarrollar objetivos de bienestar local; y por último, la quinta, establece las provisiones finales.

\section{Objetivos: desarrollo sostenible y el bienestar de las generaciones futuras}

La ley contiene numerosas provisiones y obligaciones que vinculan a los poderes públicos a llevar políticas fundamentadas en el desarrollo sostenible. La ley plantea que una noción específica de "desarrollo sostenible", que sería un proceso para alcanzar el bienestar económico, social, medioambiental y cultural, y que estaría guiado por el "principio de desarrollo sostenible" y por unas "metas de bienestar"38 establecidos en la misma ley. En este sentido, la ley diferencia el desarrollo sostenible y el principio del desarrollo sostenible. El primero sería un marco general de la política y el segundo un instrumento para llevar a cabo el primero. Esta diferenciación fortalece esta conceptualización porque no solo se enfoca en la definición estándar de desarrollo sostenible, sino que la sitúa al mismo nivel que las metas de bienestar específicas.

En este sentido, de acuerdo con esta ley el "desarrollo sostenible" es: "el proceso de mejora del bienestar económico, social, medioambiental y cultural de Gales mediante la adopción de medidas, de acuerdo con el principio de desarrollo sostenible, destinadas a alcanzar las metas de bienestar"39. El desarrollo sostenible es presentado como un proceso para mejorar el bienestar y se compone de: (1) el principio de desarrollo sostenible ${ }^{40}$ y (2) las metas de bienestar $^{41}$.

1. El principio de desarrollo sostenible: Cuando un organismo público lleve a cabo una actividad "de acuerdo con el principio de desarrollo sostenible" significa

\footnotetext{
${ }^{38}$ La ley los denomina "Well-being goals" que podrían traducirse también como "objetivos de bienestar". No obstante, como la norma incluye también los "Well-being objectives", los primeros los traduciré como "metas" y los segundos como "objetivos". Los primeros son de carácter general y son parte del "desarrollo sostenible", y los segundos son los objetivos específicos que debe marcar cada organismo público.

${ }^{39}$ Artículo 2, Well-being of Future Generations (Wales) Act 2015.

${ }^{40}$ Artículo 5, Well-being of Future Generations (Wales) Act 2015.

${ }^{41}$ Artículo 4, Well-being of Future Generations (Wales) Act 2015.
} 
que el organismo debe actuar de tal manera que busque asegurar que las necesidades de la presente generación sean satisfechas sin comprometer la capacidad de las generaciones futuras para satisfacer sus propias necesidades. Para ello, el organismo deberá (a) equilibrar las necesidades a corto plazo con la necesidad de salvaguardar la capacidad de satisfacer las necesidades a largo plazo, especialmente cuando aquello que se realice para satisfacer las necesidades a corto plazo pueda tener un efecto perjudicial a largo plazo; (b) adoptar un enfoque integrado, considerando (i) cómo los objetivos de bienestar del organismo pueden repercutir en cada una de las metas de bienestar; y (ii) cómo los objetivos de bienestar del organismo repercuten entre ellos o sobre los objetivos de otros organismos públicos (...); (c) considerar la implicación de otras personas con interés en la consecución de los objetivos de bienestar y de garantizar que esas personas reflejen la diversidad de la población en el ámbito de sus competencias; (d) justificar cómo la colaboración podría ayudar al organismo a cumplir sus objetivos de bienestar, o ayudar a otro organismo a cumplir sus objetivos; y (e) cómo el despliegue de recursos para evitar o empeorar problemas puede contribuir a la consecución de los objetivos de bienestar del organismo, o de otro organismo.

2. Las metas de bienestar: las siete metas de bienestar establecidas en la ley son: (i) prosperidad, (ii) resiliencia, (iii) salud, (iv) igualdad, (v) cohesión social, (vi) cultura y (vii) responsabilidad global. Esas metas de bienestar, además, son descritas individualmente a partir de los distintos retos que se deberán afrontar para alcanzar el bienestar: la descarbonización, la protección de la biodiversidad y los ecosistemas, el acceso a la salud y a la educación, la calidad del trabajo, la igualdad, la promoción y protección de la cultura, etcétera.

La ley entiende el desarrollo sostenible como un proceso por el cual se deben aplicar las políticas a partir del principio de desarrollo sostenible con el fin de alcanzar unas metas de bienestar determinadas. De esta forma, plantea que la políticas públicas deban llevarse a cabo teniendo en cuenta el desarrollo sostenible a partir de un doble filtro: uno como meta, las metas de bienestar, y otro como guía, el principio de desarrollo sostenible (que incluye la consideración de los impactos a largo plazo, la colaboración institucional, la adopción de un enfoque integrado, etcétera). 
A partir de este marco conceptual, la ley plantea que los distintos organismos y administraciones públicas lleven a cabo sus cometidos a través del "desarrollo sostenible", teniendo en cuenta la guía que proporciona el "principio de desarrollo sostenible" y orientándose hacia las metas de bienestar. En este proceso los organismos públicos deberán establecer y publicar aquellos objetivos de bienestar que cada una de ellas ha adoptado para (a través del desarrollo sostenible) puedan contribuir a alcanzar las metas de bienestar establecidas en la ley.

\section{Obligaciones generales: objetivos de bienestar y metas de bienestar}

Esta ley vincula a un buen número de organismos públicos. La definición de organismo público es bastante amplia: ministros, autoridades locales, organismos de protección de la naturaleza, autoridades de parques nacionales, autoridades del sistema público de salud, autoridades del sistema educativo, etcétera ${ }^{42}$. El artículo tercero establece las obligaciones generales de los organismos público, que deberán, en su ámbito competencial y territorial, (i) implementar el desarrollo sostenible; y (ii) para implementar el desarrollo sostenible deben (a) establecer y publicar los objetivos de bienestar diseñados para maximizar su contribución al logro de cada uno de los las metas de bienestar; (b) tomar todas las medidas razonables (en el ejercicio de sus funciones) para cumplir esos objetivos ${ }^{43}$.

Además, la norma prescribe cómo deben elaborarse estos objetivos de bienestar. Establece cuando los organismos públicos publiquen sus objetivos de bienestar deberán incorporar una declaración en la que expliquen (a) por qué consideran que el cumplimiento de tales objetivos contribuirá a la consecución de las metas de bienestar; (b) por qué el organismo público considera que ha establecido objetivos de bienestar de acuerdo con el principio de desarrollo sostenible; (c) las medidas que el organismo público se propone adoptar para cumplir esos objetivos de acuerdo con el principio; (d) especificar los plazos en los que el organismo espera cumplir los objetivos; y (e) proporcionar cualquier

\footnotetext{
42 Ver la lista completa en el Artículo 6, Well-being of Future Generations (Wales) Act 2015.

${ }^{43}$ Artículo 3, Well-being of Future Generations (Wales) Act 2015.
} 
otra información que el organismo considere apropiada sobre la adopción de medidas y el cumplimiento de los objetivos ${ }^{44}$.

Entre las obligaciones específicas cabe mencionar que los ministros deberán publicar indicadores e informes para poder evaluar las políticas llevadas a cabo y su grado de implementación en relación con las metas de bienestar. En este sentido los ministros deberán publicar anualmente los indicadores que deben aplicarse para medir el progreso hacia la consecución de los objetivos de bienestar. Los indicadores deben expresarse como un valor o característica que pueda medirse cuantitativa o cualitativamente con respecto a un resultado concreto. También, deberán indicar, en relación con los indicadores, los objetivos que pretenden alcanzar para así poder evaluar si se está avanzando hacia las metas de bienestar ${ }^{45}$. Los ministros, además, deberán publicar un informe que contenga predicciones de las probables tendencias futuras del bienestar económico, social, medioambiental y cultural de Gales. En la elaboración del informe deberán considerar las acciones llevadas a cabo por las Naciones Unidas en relación con los Objetivos de Desarrollo Sostenible y evaluar el impacto potencial de dicha acción en el bienestar económico, social, medioambiental y cultural de Gales, y tener en cuenta el informe que contiene una evaluación de los riesgos para el Reino Unido del impacto actual y previsto del cambio climático ${ }^{46}$. Igualmente, los ministros deberán publicar un informe sobre los progresos realizados en el cumplimiento de sus objetivos de bienestar para cada ejercicio presupuestario ${ }^{47}$.

Por último, antes entrar a analizar el Comisionado, es preciso mencionar que la aplicabilidad directa y los efectos jurídicos de la norma no son claros. En primer lugar cabe aclarar que muchos asuntos de los que se ocupa esta norma pueden ser objeto de protección jurídica ante los tribunales (como la protección ambiental, de los derechos de los trabajadores, o de la educación) y esta norma no se encarga de la protección jurídica de éstas. La cuestión es si las obligaciones que la norma impone a los organismos públicos (publicar sus objetivos, llevar a cabo acciones para alcanzar las metas de bienestar, etcétera)

\footnotetext{
${ }^{44}$ Artículo 7, Well-being of Future Generations (Wales) Act 2015.

${ }^{45}$ Artículo 10, Well-being of Future Generations (Wales) Act 2015.

${ }^{46}$ Artículo 11, Well-being of Future Generations (Wales) Act 2015.

${ }^{47}$ Artículo 12, Well-being of Future Generations (Wales) Act 2015.
} 
pueden ser exigidos ante los tribunales por los ciudadanos. Una buena parte del articulado está redactado en términos de obligación (utilizando en muchas ocasiones "must" y no "shall"). Por ello, considero que algunos aspectos pueden generar obligaciones directas a los organismos públicos, como la obligación de publicar los objetivos de bienestar (the action a public body takes in carrying out sustainable development must include (a) setting and publishing objectives...); pero, contrariamente, el contenido de dichos objetivos no serían enjuiciables (...and taking all reasonable steps to meet these objectives). Así, por un lado los organismos deberán publicar los objetivos, pero sus políticas en torno a éstos no serán enjuiciables. En este sentido, de acuerdo con la interpretación de Davies la ley no impone deberes exigibles ante los tribunales ${ }^{48}$.

En este sentido, la ley incluye un control externo a través del Auditor General de Gales que sería el encargado de examinar las actividades llevadas a cabo por los organismos públicos y evaluar si ha actuado de acuerdo con el principio de desarrollo sostenible a la hora de establecer sus objetivos de bienestar y ha adoptado medidas adecuadas para cumplir con dichos objetivos ${ }^{49}$.

\section{Comisionado para el Bienestar de las Generaciones Futuras}

El Comisionado se presenta como una figura independiente que debe evaluar el trabajo llevado a cabo por otras instituciones y organismos en relación a las metas y los objetivos de bienestar. No obstante, esta independencia es cuestionable. El Comisionado es elegido por los ministros galeses en consulta con la Asamblea Nacional (ahora Parlamento) a través del comité responsable. Su mandato dura siete años y podrá ser destituido por los ministros si consideran que no es apto para continuar o no puede o no quiere ejercer sus funciones. Además, a pesar de que podrá contratar personal, los ministros deberán aprobar la cantidad de personal contratado y sus condiciones contractuales. Aquí radica una cuestión que puede ser problemática. Así, por un lado, su nombramiento y destitución están supeditadas a la decisión del gobierno y, por otro lado, su capacidad para auditar a las diferentes instituciones y organismos queda vinculada con su capacidad económica que depende directamente del ejecutivo.

\footnotetext{
${ }^{48}$ Vid. Davies, H., "The Well-being of Future Generations (Wales) Act 2015 - A Step..." Op. Cit. p. 175.

${ }^{49}$ Artículo 15, Well-being of Future Generations (Wales) Act 2015.
} 
Los deberes principales son promover el principio de desarrollo sostenible, actuar para proteger las capacidades de las generaciones futuras para satisfacer sus necesidades, y fomentar que los organismos públicos tengan en cuenta el impacto a largo plazo de las políticas que lleven a cabo. Para ello, deberá supervisar y evaluar el grado de cumplimiento de los objetivos fijados por los organismos públicos ${ }^{50}$.

En cuanto a sus funciones, son variadas y podrían dividirse entre aquellas de asesoramiento y asistencia; y las de control, auditoria, y recomendaciones.

Entre las primeras: asesorar a cualquier organismo público (incluyendo asesoramiento sobre el cambio climático); asesorar al Auditor General de Gales sobre el principio de desarrollo sostenible; asesorar a las juntas de servicio público a preparar sus planes de bienestar local; asesorar a cualquier otra persona que el Comisario considere que está tomando (o desea tomar) medidas que puedan contribuir a la consecución de las metas de bienestar; fomentar las mejores prácticas entre los organismos públicos a la hora de tomar medidas para cumplir sus objetivos de bienestar de acuerdo con el principio de desarrollo sostenible; o promover la concienciación entre los organismos públicos sobre la necesidad de tomar medidas para cumplir sus objetivos de bienestar de acuerdo con el principio de desarrollo sostenible ${ }^{51}$.

Entre las segundas: el Comisionado puede evaluar e investigar la coherencia de las metas de bienestar y los indicadores nacionales con el principio de desarrollo sostenible; evaluar si se tiene en cuenta el principio de desarrollo sostenible en los indicadores nacionales; dar cuenta del propio principio de desarrollo sostenible (incluyendo cómo se aplica para establecer y cumplir los objetivos); y cualquier actividad que esté vinculada con bienestar económico, social, medioambiental y cultural de Gales (o de cualquier parte de Gales) ${ }^{52}$. Además, también podrá emitir recomendaciones a los organismos públicos sobre los pasos que ha llevado a cabo o ha propuesto para alcanzar sus objetivos de bienestar ${ }^{53}$, y a los ministros sobre las metas de bienestar y los indicadores

\footnotetext{
50 Artículo 18, Well-being of Future Generations (Wales) Act 2015.

51 Artículo 19.1, Well-being of Future Generations (Wales) Act 2015.

52 Artículo 19.2, Well-being of Future Generations (Wales) Act 2015.

${ }^{53}$ Artículo 20.4, Well-being of Future Generations (Wales) Act 2015.
} 
nacionales ${ }^{54}$. Estas recomendaciones no son vinculantes ${ }^{55}$, pero la norma indica que los organismos públicos deberán llevar a cabo medidas para seguir las recomendaciones hechas por el Comisionado.

Además, el Comisionado debe preparar y publicar un informe para cada periodo sobre las mejoras que los organismos públicos deben realizar para cumplir con los objetivos de bienestar y en aplicación del principio de desarrollo sostenible. El informe incluirá una evaluación de cómo los organismos públicos deberían (a) salvaguardar mejor la capacidad de las generaciones futuras para satisfacer sus necesidades, y (b) tener más en cuenta el impacto a largo plazo en las actividades realizadas.

En el año 2020 publicó su primer informe. La primera característica que se debe destacar es la facilidad y publicidad de la información relativa a su trabajo. El Comisionado dispone de una página web en la que se puede navegar con suma facilidad y es posible analizar el informe desde un sistema interactivo que puede ser leído o escuchado ${ }^{56}$. Además, también, el informe tiene la opción de ser leído en formato libro, pero que también incluye imágenes, vídeos y audios ${ }^{57}$. informe establece una pregunta clave ¿Qué Gales queremos en 2050? A partir de esta pregunta, desarrolla un análisis sobre las siete metas de bienestar y las analiza con una perspectiva a largo plazo. En este análisis informa de las debilidades y riesgos que se observan a medio y largo plazo: el cambio climático, la desigualdad, el cambio de modelo de transporte, la descarbonización, el sistema sanitario, la protección a la infancia, el acceso a la vivienda, entre otros. Para cada una de ellas también propone alternativas y orienta a los organismos sobre dónde podrían incidir para mejorar sus objetivos y alcanzar las metas de bienestar. Las recomendaciones no solo se vinculan con lo que deberían llevar a cabo, sino también qué actividades deberían cesar. Así, indica qué políticas deberían cesar para alcanzar los objetivos y qué políticas deberían impulsarse. También se hace eco de los posibles cambios en el futuro en relación con la demografía, el medio ambiente, los recursos naturales, y los problemas de

\footnotetext{
${ }^{54}$ Artículo 21, Well-being of Future Generations (Wales) Act 2015.

${ }^{55}$ Artículo 22, Well-being of Future Generations (Wales) Act 2015

56 Cfr. "Future Generations Report 2020". [Disponible aquí: https://www.futuregenerations.wales/wp-content/uploads/2020/05/FGC-Report-English.pdf. Última consulta el 4 de abril de 2021]

57 Ídem.
} 
desigualdad. En relación a éstos, también hace referencia a los cambios vinculados con los trabajos que se desarrollarán en el futuro y el cambio en los conocimientos y habilidades que tendrán mayor valor en el futuro. Esta predicción la vincula con el futuro del mercado de trabajo, el sistema productivo y el sistema educativo y universitario.

Resulta de interés, además, que el informe no solo se fundamenta en un estudio técnico en el que recoge un análisis de las diferentes metas y retos de cara al futuro, sino que también recoge la percepción de la ciudadanía sobre estas metas y retos. Así, no solo informa a las administraciones públicas de aquello que deberían mejorar con respecto a las metas de bienestar, sino que informan de aquello que opinan los ciudadanos sobre su implementación.

\section{ANÁLISIS Y CONCLUSIONES}

Esta ley proporciona herramientas que pueden ayudar a contrarrestar los problemas del cortoplacismo político y la inconsistencia temporal de las políticas públicas.

En primer lugar, se estructura en torno al concepto de bienestar. No solo en el bienestar de las generaciones futuras, sino en el bienestar en general, que incluye bienestar económico, social, ambiental y cultural. La ley comprende el desarrollo sostenible como un proceso para alcanzar el bienestar a partir del reconocimiento de los límites ambientales existentes. De esta forma considero que puede ser más fácil vincular a las generaciones presentes con las futuras. Las metas de bienestar no solo beneficiarían a las generaciones futuras, sino que establecerían un vínculo entre generaciones. Pero este vínculo no se fundamenta en una responsabilidad entre grupos de generaciones estáticas y separadas en el tiempo, en el que la presente se responsabiliza con aquellas que vivirán en un futuro remoto. El vínculo que establece es entre las diferentes generaciones que con el paso del tiempo se van entrelazando y, a través de una noción de desarrollo sostenible fundamentada en el bienestar, promueve políticas que deberían mejorar el bienestar presente sin comprometer el de las generaciones venideras. Esto es, que la búsqueda del bienestar y su sostenibilidad en el tiempo deben estar en el centro de la acción política. En este sentido, el doble filtro establecido en la noción de "desarrollo sostenible" que, por un lado, plantea unas metas de bienestar concretas y, por otro, el principio de 
desarrollo sostenible, que plantea la necesidad de llevar a cabo políticas a largo plazo, incluyendo unos objetivos específicos, puede ser determinante para que las instituciones eviten la tentación cortoplacista.

En segundo lugar, la ley establece una responsabilidad compartida entre muchas instituciones. No solo pone el peso en una institución determinada, sino que institucionaliza el bienestar intergeneracional en todos los organismos públicos. De este modo, no solo existirá una institución determinada que deba hacer frente a los problemas vinculados con las generaciones futuras, sino que todas las instituciones deben incluir sus objetivos para lograr dichas metas de bienestar y la aplicación del principio de desarrollo sostenible. En este sentido, la descentralización de responsabilidades puede generar mayor aceptación e, incluso, podría ser determinante para que la ciudadanía demande más acción a sus representantes. Esto es, como cada organismo público (universidades, entes locales, sistema de salud, etcétera) deberá establecer sus objetivos de bienestar, es más fácil para la ciudadanía y las organizaciones no gubernamentales acceder a este tipo de instituciones y exigir mayor ambición en sus políticas públicas para alcanzar las metas de bienestar. Además, la misma ley incorpora la posibilidad de que la ciudadanía se sume a la elaboración de proyectos vinculados con los objetivos de bienestar de las distintas instituciones.

En tercer lugar, a partir del planteamiento anterior, la norma crea un mecanismo de control informal en el que la ciudadanía puede examinar el trabajo llevado a cabo por los organismos públicos, ya que éstos tienen la obligación de publicar los distintos los objetivos de bienestar y de justificar cómo éstos servirán para alcanzar las metas de bienestar. Además, los ministros deberán hacer públicos los indicadores que aportarán información de carácter cualitativo y cuantitativo, y deberán presentar sus informes anuales sobre sus actividades. Esto hace que las organizaciones no gubernamentales, los movimientos sociales y la ciudadanía puedan controlar mejor a los organismos públicos en relación con sus políticas de bienestar. Así, es posible un control ciudadano desde diferentes escalas y ámbitos de acción política.

En cuarto lugar, la creación del Comisionado tiene ciertamente aspectos muy positivos. Parece que su mandato está limitado competencialmente y no tiene poder para vetar políticas públicas, ni de recurrir políticas o legislación ante los 
tribunales. No obstante, su capacidad para auditar, asesorar y publicar un informe sobre las actividades no es baladí. Esta institución puede canalizar toda la información de todos los organismos públicos para dar cuenta de las proyecciones del país a medio y largo plazo. El Comisionado, además de auditar, asesorar e informar, genera un conocimiento que puede ser muy relevante para analizar la orientación de las políticas públicas en términos de bienestar y desarrollo sostenible.

Desde una perspectiva comparada, el actual Defensor de las Generaciones Futuras de Hungría, Gyula Bándi, se hizo eco de los avances en materia ambiental en Hungría desde la reforma constitucional que incluyó la referencia a las generaciones futuras e instituyó la figura que él representa en la actualidad ${ }^{58}$. En su texto, el profesor Bándi señala que el trabajo del Defensor de las Generaciones Futuras ha ayudado a incorporar en la esfera pública los planteamientos a largo plazo. Así, a pesar de que la institución y con diferentes cometidos $^{59}$, como afirma Bándi, su misión principal es recordar al Estado, en todos sus órganos y niveles, sus deberes y responsabilidades en torno a las generaciones futuras ${ }^{60}$. En este sentido, Bándi da cuenta de la creciente relevancia que tiene la institución en el trabajo del Tribunal Constitucional. Es preciso destacar que éste incorporó recientemente los argumentos que el Defensor presentó en un amicus curiae sobre los impactos de carácter intergeneracional en la modificación de la legislación relativa a la gestión de aguas $^{61}$. Así, en un sentido análogo la influencia del Comisionado, a pesar de que su rol es eminentemente político, puede sobrepasar el ámbito político y fortalecer la interpretación del concepto de desarrollo sostenible en su aplicación jurídica.

Es preciso, destacar algunas dificultades inherentes al Comisionado. La dependencia directa del poder político puede repercutir en su capacidad de fiscalización y crítica de éste. El hecho que sus poderes estén supeditados al

\footnotetext{
58 Vid. Bándi, G., "Interests of Future Generations, Environmental Protection and the Fundamental Law", Journal of Agricultural and Environmental Law, Vol. 15, No. 19, 2020.

${ }^{59}$ Para un análisis de esta institución, véase, Jávor, B., "Institutional Protection of Succeeding Generations: Ombudsman for Future Generations in Hungary", en Tremmel, J. C. (Ed.), Handbook of Intergenerational Justice, Edward Elgar Publishing, 2006, pp. 282-98.

${ }^{60}$ Vid. Bándi, G., "Interests of Future Generation... Op. Cit., p. 10,

${ }^{61}$ Ibíd., pp. 18-19.
} 
poder político puede disminuir su capacidad de fiscalización a los organismos públicos y que su independencia se vea afectada. Además, esta dependencia también se hace palpable al hecho de que el presupuesto y la contratación de personal dependan directamente del poder político, lo que puede limitar su capacidad de influencia y análisis de las políticas llevadas a cabo.

En quinto lugar, considero, que la ley reúne en un mismo texto más de una herramienta, mecanismo e institución para afrontar el problema del cortoplacismo político y poder llevar a cabo políticas a largo plazo. Así, elimina lo desincentivos electoralistas de los políticos, pues incluye herramientas que obligan a considerar el largo plazo, e involucra a la ciudadanía en la toma de decisiones entorno al bienestar. En el mismo sentido, en las limitaciones del Comisionado para influir decididamente en las políticas públicas late una de sus fortalezas, pues su carácter contramayoritario no generará problemas de legitimidad política y puede favorecer a un mejor diálogo entre instituciones y sociedad civil. Como no, este carácter débil puede llevar a que los organismos no sean diligentes con la presentación de sus objetivos de bienestar ni que lleven a cabo políticas ambiciosas en torno a las metas de bienestar. En este sentido, también dependerá de la capacidad del Comisionado y, como no, el interés de la sociedad civil y los representantes públicos. Es preciso mencionar que la aplicación de las políticas no solo dependerá de la implicación de los representantes políticos y el trabajo del Comisionado, sino también de la implicación de la sociedad civil en la consecución de éstos por parte de las diferentes administraciones.

Esta norma aporta instrumentos realmente interesantes a la vida política de Gales. El hecho de que sitúe en el centro de la vida política el bienestar, la sostenibilidad y la cohesión social, vincula a las instituciones y organismos para que lleven a cabo políticas en este sentido y tengan en cuenta estos elementos para el mismo ejercicio de su cargo. La publicidad, el acceso a la información y la apertura hacia la sociedad civil facilitan la participación pública en la toma de decisiones. Esta interacción puede ser positiva para mejorar la deliberación de asuntos de gran relevancia. Así, como he sostenido, los asuntos que requieren una mirada a largo plazo deben ser continuos y esta apertura a la participación puede ayudar a que las políticas sean constantes a lo largo del tiempo. Por 
último, el Comisionado es central para concretar el proceso de deliberación pública sobre las metas de bienestar. Su capacidad fiscalizadora facilita a publicitar los desafíos que la sociedad tiene por delante y el trabajo que las administraciones llevan a cabo. Esta capacidad de vincular la política actual con los retos intergeneracionales es central para que la ley ayude a mejorar la toma de decisiones en aspectos centrales de la vida política actual y que afectarán de manera decisiva en el futuro.

\section{BIBLIOGRAFÍA}

Bándi, G., "Interests of Future Generations, Environmental Protection and the Fundamental Law", Journal of Agricultural and Environmental Law, Vol. 15, No. 19, 2020.

Bidadanure, J., "Youth Quotas, Diversity, and Long-Termism: Can Young People Act as Proxies for Future Generations?", en González-Ricoy, I., y Gosseries, A., (eds), Institutions for Future Generations, Oxford University Press, 2016.

Capella, J. R., Un fin del mundo: Constitución y democracia en el cambio de época, Ed. Trotta, Madrid, 2019

Courtis, C., "La prohibición de regresividad en materia de derechos sociales: apuntes introductorios", en Courtis, C., (Comp.): Ni un paso atrás. La prohibición de regresividad en materia de derechos sociales, Buenos Aires, Centro de Estudios Legales y Sociales (CELS) y Centro de Asesoría Legal del Perú (CEDAL). Ediciones del Puerto, 2006.

Davies, H., "The Well-being of Future Generations (Wales) Act 2015 - A Step Change in the Legal Protection of the Interests of Future Generations?", Journal of Environmental Law, 29, 2017

Dobson, A., "Representative Democracy and the Environment", en Lafferty, W. M., y Meadowcroft, J., (Eds.), Democracy and the Environment: Problems and Prospects, Cheltenham, Edward Elgar. 1996.

Ekeli, K. S., "Giving a Voice to Posterity - Deliberative Democracy and Representation of Future People", Journal of Agricultural and Environmental Ethics, 18(5), 2005.

Ekeli, K. S., "Green Constitutionalism: The Constitutional Protection of Future Generations", Ratio Juris, Vol. 20 No. 3, 2007, pp. 378-401.

Ferrajoli, L., Derechos y garantías: la ley del más débil, Ed. Trotta, (8ª ed.), 2016. [Traducción de Ibáñez, P. A., y Greppi, A.]

Ferrerres Comella, V., Justicia constitucional y democracia, Centro de Estudios Políticos y Constitucionales, (2ed.), 2007.

Ferrerres Comella, V., Constitutional Courts Democratic Values: a European perspective, Yale University Press, New Haven and London, 2009.

González-Ricoy, I., "Instituciones intergeneracionales y cortoplacismo político", Revista Española de Ciencia Política, 41, 2016. 
González-Ricoy, I., y Gosseries, A., (Eds.), Institutions for Future Generations, Oxford University Press, 2016.

Gosseries, A., y Meyer, L. H., (Eds.), Intergenerational justice, Oxford University Press, 2009.

Häberle, P., "Un derecho constitucional para las futuras generaciones. La otra reforma del contrato social: el contrato generacional", Lecciones y Ensayos, Número 87, Enero-Diciembre, 2009. [Traducción de Fellay, M., y Ferreyra, L.].

Jávor, B., "Institutional Protection of Succeeding Generations: Ombudsman for Future Generations in Hungary", en Tremmel, J. C. (Ed.), Handbook of Intergenerational Justice, Edward Elgar Publishing, 2006, pp. 282-98.

Luiselli, V., Lost Children Archive, Alfred A. Knopf, 2019.

Meyer, L. H., Intergenerational justice, Routledge, 2012.

Nogueira, A., "La huella ecológica. El establecimiento de indicadores ambientales y su significación para el Derecho", Revista Catalana de Dret Ambiental, Vol. 10, Núm. 1, 2019.

Pontara, G., Ética y generaciones futuras, Ariel, Barcelona, 1996.

Riechmann, J., Un mundo vulnerable, Los Libros de la Catarata, Madrid, 2005 ( $2^{\mathrm{a}}$ ed.).

Roemer, J., y Suzumura, K. (Eds.), Intergenerational Equity and Sustainability, Palgrave Macmillan, 2007.

Sikora, R.I., y Barry, B. (Eds.), Obligations to Future Generations, Philadelphia, Temple University Press, 1978.

Thompson, D. F., "Representing future generations: political presentism and democratic trusteeship", Critical Review of International Social and Political Philosophy, 13 (1), 2010.

Tremmel, J. C., (Ed.) Handbook of Intergenerational Justice, Edward Elgar, 2006.

Waldron J., Law and Disagreements, New York, Oxford University Press, 1999.

Waldron, J., "The Core of the Case against Judicial Review", Yale Law Journal, 115, 2006. 